\title{
Obstetric fistulae in southern Mozambique: incidence, obstetric characteristics and treatment
}

Sibone Mocumbi ${ }^{1,2^{*}}$ (D), Claudia Hanson ${ }^{3,4}$, Ulf Högberg ${ }^{2}$, Helena Boene ${ }^{5}$, Peter von Dadelszen ${ }^{6}$, Anna Bergström ${ }^{2,7}$, Khátia Munguambe ${ }^{5,8}$, Esperança Sevene $e^{5,9}$ and the CLIP working group

\begin{abstract}
Background: Obstetric fistula is one of the most devastating consequences of unmet needs in obstetric services. Systematic reviews suggest that the pooled incidence of fistulae in community-based studies is 0.09 per 1000 recently pregnant women; however, as facility delivery is increasing, for the most part, in Africa, incidence of fistula should decrease. Few population-based studies on fistulae have been undertaken in Sub-Saharan Africa, including Mozambique. This study aimed to estimate the incidence of obstetric fistulae in recently delivered mothers, and to describe the clinical characteristics and care, as well as the outcome, after surgical repair.

Methods: We selected women who had delivered up to 12 months before the start of the study (June, 1st 2016). They were part of a cohort of women of reproductive age (12-49 years), recruited from selected clusters in rural areas of Maputo and Gaza provinces, Southern Mozambique, who were participating in an intervention trial (the Community Level Interventions for Pre-eclampsia trial or CLIP trial). Case identification was completed by self-reported constant urine leakage and was confirmed by clinical assessment. Women who had confirmed obstetric fistulae were referred for surgical repair. Data were entered into a REDCap database and analysed using R software.

Results: Five women with obstetric fistulae were detected among 4358 interviewed, giving an incidence of 1.1 per 1000 recently pregnant women $(95 \% \mathrm{Cl} 2.16-0.14)$. All but one had Caesarean section and all of the babies died. Four were stillborn, and one died very soon after birth. All of the patients identified and reached the primary health facility in reasonable time. Delays occurred in the care: in diagnosis of obstructed labour, and in the decision to refer to the secondary or third-level hospital. All but one of the women were referred to surgical repair and the fistulae successfully closed.

Conclusion: This population-based study reports a high incidence of obstetric fistulae in an area with high numbers of facility births. Few first and second delays in reaching care, but many third delays in receiving care, were identified. This raises concerns for quality of care.
\end{abstract}

Keywords: Obstetric fistula, Caesarean, Incidence, Population-based, Sub-Saharan Africa

\footnotetext{
* Correspondence: sibone.mocumbi@gmail.com; sibone.mocumbi@kbh.uu.se

'Department of Obstetrics and Gynaecology, Faculty of Medicine,

Universidade Eduardo Mondlane (UEM), Av. Agostinho Neto 679, 1100

Maputo, Mozambique

2Department of Women's and Children's Health, Uppsala University,

Akademiska sjukhuset, SE-75185 Uppsala, Sweden

Full list of author information is available at the end of the article
} 


\section{Plain English summary}

Obstetric fistula is a childbirth complication in which a hole develops between the bladder and vagina or between the rectum and vagina. It occurs after a prolonged and neglected labour without adequate assistance or might happen as a complication after caesarean delivery, and causes a constant leaking of urine and/or faeces through the vagina.

There is no reliable data in Mozambique about the number of women suffering this preventable condition. This study was to estimate the number of women who developed obstetric fistula during the year before the start of the study (incidence), describe their clinical characteristics and the results of the surgical repair. Women with obstetric fistula were identified by self-reported constant urine leakage and the condition confirmed by physician's examination.

Women who delivered in rural areas of Southern Mozambique, 12 months before June, 1st 2016, were interviewed about their delivery experiences, birth outcomes, complications and satisfaction with care. Among 4358 interviewed, five presented obstetric fistula, giving an incidence of 1 woman developing obstetric fistula for each 1000 recently pregnant women. The study shows that all women diagnosed with fistula, had arrived at the health centre in time. All but one had a caesarean delivery. Delays were identified in attendance, referral and care. Afterwards, all had successful fistulae's surgical repair.

The conclusion was that the incidence of obstetric fistula is high in an area with a high facility-based delivery rate. We are concerned about the quality of care as many of the fistula cases occurred in the health facilities.

\section{Background}

Obstetric fistula is one of the most devastating consequences of unmet needs in obstetric services. The World Health Organization (WHO) defines a fistula as an abnormal opening between the woman's vagina and bladder and/or rectum, through which her urine and/or faeces continually leak [1]. Estimates indicate that two million women suffer from undetected or untreated fistulae globally, and that 50,000 to 100,000 new cases occur each year, mainly in low- and middle-income countries (LMICs) in sub-Saharan Africa and South Asia [2], where maternal mortality is high $[3,4]$. While the most common cause of fistula is prolonged or obstructed labour $[5,6]$, recent studies suggest that an increasing proportion of urogenital fistulae in LMICs may be iatrogenic, mainly resulting from caesarean section [5].

Reported rates of obstetric fistula in LMICs vary widely, from 0 to 4.09 obstetric fistula cases per 1000 deliveries, and reliable data on the prevalence and incidence are sparse [7]. Most estimates are based on self-reporting, personal communication with surgeons, studies conducted by advocacy groups, and the review of hospital series. In all of these reports, the relevant denominators, such as the source population and number of births, are unknown or unreported [8-10]. Only six studies, all from LMICs, were judged to be of sufficient quality for a systemic review by Adler et al. (2013) [11]: three community-based studies [12-14]; and three hospitalbased studies [15-17]. The pooled incidence of fistula ranged from 0.09 per 1000 recently pregnant women in the community-based studies to 0.66 per 1000 recently pregnant women in the hospital-based studies. The review highlighted that the included studies had important methodological limitations: most being retrieved populationbased studies, which were not included in the estimate, and reported on self-reported occurrence of fistulae without physical examination by a trained healthcare provider. Alternatively, studies where obstetric fistulae were clinically confirmed, were primarily derived from hospitals, thus lacking a denominator and detailed information of the population from where the women originated.

Despite being more expensive, community-based surveys generally provide wider coverage, better representation of a regional or national population, and more opportunities to collect a wide range of data compared to facility-based studies [18]. However, without clinical examination, such studies cannot establish accurate disease rates, and making a correct diagnosis without eliminating other causes of incontinence is not possible $[7,19,20]$.

Obtaining reliable epidemiologic data on fistula is important to efficiently direct programme efforts [1, 21]. In Mozambique there is an on-going programme for fistula prevention and treatment, which consists of raising awareness, early detection and surgery campaigns in all provinces, however, exact data on the prevalence and incidence are not available [22], thus limiting the effectiveness of programme planning [23]. In 2015, it was estimated that 100,000 women suffer from obstetric fistula in Mozambique [22], and only 556 were identified and underwent surgery for the condition during the campaigns for fistula treatment [Melo, A.; Unpublished data from the Mozambican National Programme for fistula prevention and treatment, 2015].

The objective of the current study was to describe the problem of obstetric fistulae in rural areas of Southern Mozambique. The specific aims were: to estimate the incidence of obstetric fistulae in recently delivered mothers, to describe the women's obstetric profile and their self-reported health care, as well as to report outcomes after surgical repair.

\section{Methods}

The study design was a survey within a population cohort. We interviewed recently delivered women (mothers), 
defined as those having given birth during the 12 months before the start of the study (June, 1st 2016). We did not, however, include those who had delivered in the two weeks preceding this time, as fistula usually occurs between one and 10 days after delivery. These mothers were identified within a cohort of women of reproductive age (12-49 years) from the ongoing Community Level Interventions for Pre-eclampsia (CLIP) trial (NCT01911494) [24], covering households in rural areas of Maputo and Gaza provinces, Southern Mozambique. The CLIP database was only used to facilitate the identification of the women and their households. We identified self-reported symptoms of fistulae and any suspected fistulae were confirmed by clinical assessment.

An obstetric fistula was defined as an abnormal opening between the woman's vagina and bladder and/or rectum, through which her urine and/or faeces continually leak. The fistula incidence was calculated using the total number of women who delivered as the denominator.

\section{Study setting and participants}

The study area included 12 rural clusters of Maputo and Gaza provinces in southern Mozambique, with an estimated population of 72,150 women of reproductive age and 17,400 births per year. The proportion of births attended by skilled health personnel in the study area is $85 \%$, and the caesarean section rate is at 3.8\% [Sacoor, C.; Unpublished data from CISM Demographic Surveillance, Manhiça, 2017].

The area is served by 32 health centres, providing essential preventive and curative services, which include basic antenatal care and assistance with normal births. Complicated cases are referred to the secondary level health facilities, which include four rural and one district hospital conducting routine surgical interventions, such as caesarean sections or obstetric hysterectomies, and which have larger diagnostic capacity. Two Provincial Hospitals provide tertiary-level care and one Central Hospital provides specialized care (quaternary level).

The sample size calculation was based on the prevalence of $0.15 \%$ reported by Adler et al. [11]A sample of 3700 births was deemed appropriate to estimate the prevalence with 0.05 significance level.

A list of 4441 recently pregnant women (mothers) was produced. Births (live and stillbirth) were approximated with equal numbers of pregnancies. We interviewed all mothers who we were able to recruit after a maximum of two attempts and who agreed to participate in the study.

Local authorities, including traditional leaders and village heads, were informed about the objectives of the study; a mobilization process that was important to ensure the participation of the mothers.

\section{Study instrument}

The study instrument was part of a bigger study; an assessment of mothers' experiences and birth outcomes. We used a questionnaire sequence aimed at women who had delivered a baby in the past year to collect information on their place of residence, place of birth, birth outcomes, childbirth complications, morbidity (including fistulae) and satisfaction with care, including incidences of disrespect and abuse during childbirth. Questions addressing fistulae were adapted from the United Nations Population Fund (UNFPA) proposal on obstetric fistula for the existing Demographic and Health Survey (DHS) fistula module questionnaire [25]. Information on birth outcomes was also retrieved from the antenatal and perinatal card (Caderneta de Saúde da Mulher). Mothers' birth experiences and perceived health care responsiveness were measured by adapting selected questions from the World Health Organisation (WHO) Health System Responsiveness Questionnaire [26] and the Maikhanda study undertaken in Malawi [27, 28]. The questions were translated from English to Portuguese, and the questionnaire was pre-tested and piloted before application to ensure that the questions were clear and understandable. The questionnaire was programmed to be used on a tablet using ODK Collect version 1.4.6 [29, 30]. Clinical data from the mothers with suspected fistulae were collected using a standard form. An in-depth interview guide was used to collect the narratives of the mothers with confirmed fistula. The mothers' questionnaire can be found in Additional file 1.

\section{Data collection}

Data were collected by means of structured interviews at household level between June 1st, 2016 and October 28th, 2016 by 13 experienced female data collectors who had been trained on the protocol, the data collection forms, and the administration of informed consent. Special focus was placed on the appropriate approaches to take when asking sensitive questions and on when to communicate the Portuguese questions in the local language (Changana). Data collectors were monitored by the field supervisor to ensure their compliance with the study protocol. The supervisors performed random second interviews with $1 \%$ of the women to test the quality of the data and to determine whether the data collectors needed re-training. Once a week, the PI and the data management team reviewed both the completed questionnaire and the database to check for missing answers, duplications and inconsistencies, and, if needed, the data collector was sent back to the field to gather data where corrections and clarifications were necessary.

All interviewed mothers who responded 'Yes' to the question 'Have you, AFTER your last pregnancy, ever experienced a constant leakage of urine or stool from 
your vagina during the day and night?' were re-visited at home to be invited to participate and to schedule a day for clinical examination and appropriate diagnosis at the Manhiça District Hospital. A gynaecologist (SM) completed a standardised history and clinical examination. The diagnosis of fistula was confirmed by observation of urine leakage and clear visualization of the fistula. A dye test, with methylene blue, involved back-filling the bladder and temporarily occluding the urethra for evaluation of dye leakage. We used the Waaldijk classification $[21,31,32]$. Mothers with confirmed fistula were invited to provide an in-depth interview (IDI) to provide narratives of their experiences, which will be analysed and reported elsewhere. Information on the duration of labour was collected from the IDI and used for classification of first, second and third delays in birth attendance [33].

All mothers identified as having a fistula were referred to Maputo Central Hospital for surgical fistula repair. The study team provided transport and support throughout the process. Continence after surgical repair was assessed by asking about any involuntary urine leakage and by conducting a cough test.

\section{Data management and analysis}

All data were electronically captured on-site and uploaded weekly to the Manhiça Health Research Center (CISM) database using REDCap version 6.14.0 (Vanderbilt University 2016) [34]. Database content was checked for missing answers, duplications and inconsistencies. Data were then exported to $\mathrm{R}$ software (version 3.3.1) for further analysis [35].

The incidence proportion, with $95 \%$ confidence interval, was determined by calculating the number of confirmed fistula cases as a proportion of the surveyed mothers who had given birth within 1 year prior to the interview. Descriptive statistics for each woman were obtained from the database.

\section{Results}

\section{Incidence of fistula}

Of all mothers registered as having delivered in the 12 months prior to the start of the study, 4358 were interviewed and only 18 did not agree to participate (Fig. 1). Out of all mothers, $10.4 \%$ delivered at home, $2.4 \%$ delivered on the road to a health facility, and $87.2 \%$ delivered in facilities. Of the facility deliveries, $0.5 \%$ had ventouse, and $3.6 \%$ had caesarean section.

Out of the 30 mothers who reported a constant leakage of urine, two did not agree to be examined, and 28 went on to attend at the Manhiça District Hospital for clinical examination by the gynaecologist. Six mothers were diagnosed as having obstetric fistula and were referred for surgical repair. The pre-surgery examination confirmed obstetric fistula (all vesico-vaginal) in 5 mothers.

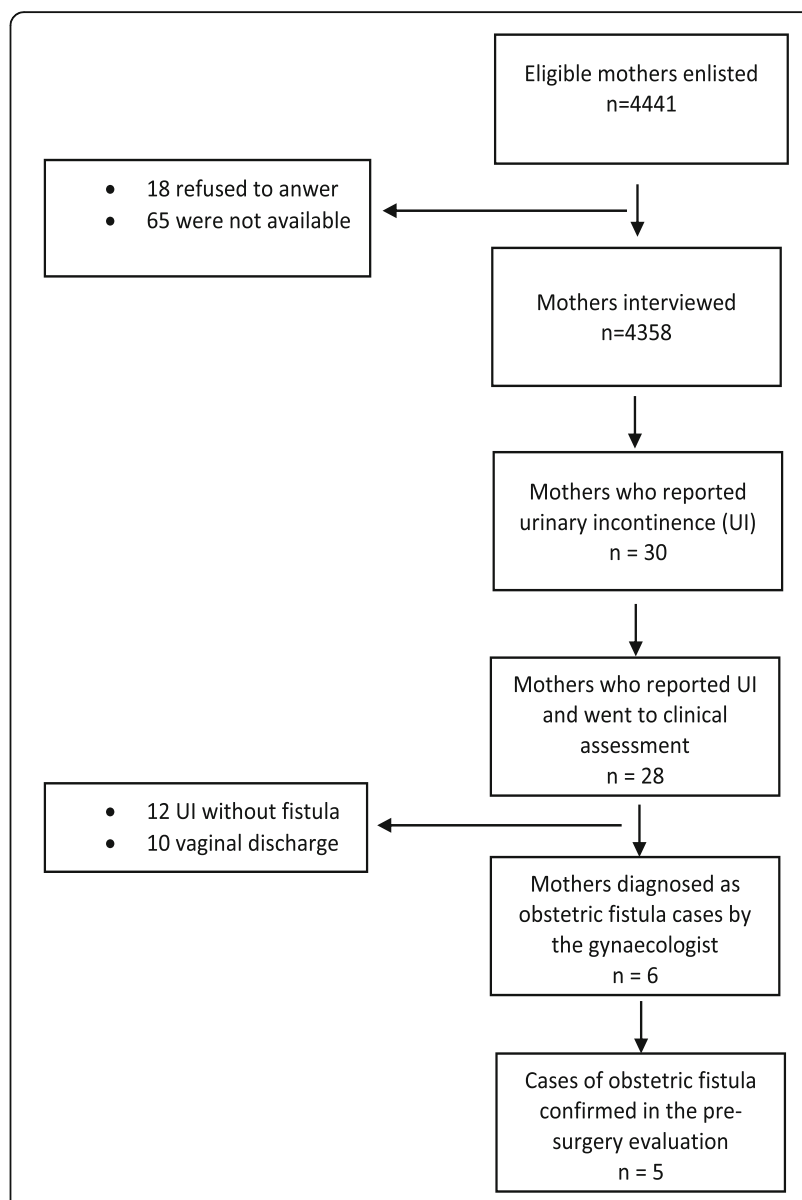

Fig. 1 Diagram showing the flow through the study

The sixth mother had severe urinary incontinence, misdiagnosed as intra-cervical fistula. From the remaining examined mothers, urinary incontinence was confirmed in twelve cases, and ten were in fact vaginal discharge without urinary incontinence. Thus, in total, five cases of vesicovaginal fistulae were diagnosed out of 4358 mothers interviewed. We estimated the fistulae incidence among recently pregnant women to be at 1.1 per 1000 recently pregnant women (95\% CI 2.16-0.14).

During the study's implementation, a further nine women came voluntarily to the study hospital seeking care for fistula-like symptoms. These were observed and a diagnosis of fistula confirmed, but the women were not included in the study because the fistula happened more than one year before the study began. All were referred to the Central Hospital for surgery.

\section{Obstetric and clinical characteristics of the women affected by fistula}

The characteristics of the five mothers diagnosed with recent obstetric fistulae are given in Table 1 . The median age was 21 years, and four were aged under 24 years. Three were primiparas. Two had been identified has 
Table 1 Background, perinatal characteristics and outcome of the five mothers diagnosed with obstetric fistula in Maputo and Gaza provinces, Mozambique 2016

\begin{tabular}{ll}
\hline Characteristics & Fistula $(n=5)$ \\
\hline Age (years) & 2 \\
$15-19$ & 3 \\
$20-29$ & \\
Education & 1 \\
No education & 4 \\
Primary & \\
Parity & 3 \\
One birth & 2 \\
Two to fourth births & \\
Previous stillbirth & 0 \\
Yes & 5 \\
No & \\
Previous neonatal death & 1 \\
Yes & 4 \\
No & \\
Mode of delivery & \\
Vaginal & \\
Caesarean & \\
Perinatal outcome & \\
Neonatlirth & 1 \\
\hline
\end{tabular}

having a height of less than $150 \mathrm{~cm}$ during the antenatal consultations, but did not receive special attention regarding this obstetric risk. All five reported seronegative HIV status.

The fistula characteristics are presented in Table 2. Three were type I fistula; the other two were: types II A and $B$. An assessment of the probable mechanism of the fistula (ischaemic, iatrogenic or combination) is presented for each participant.

\section{Patient-reported experiences of labour}

The mothers' decision to seek care was made in a timely manner, between a minimum of $1 \mathrm{~h}$ and a maximum of $7 \mathrm{~h}$. All used a private car or taxi to reach the primary health care facility and took no more than $6 \mathrm{~h}$ to arrive. Regarding the deliveries (Table 2), all reported having been referred from the primary health care facility to the secondary hospital. Labour duration, estimated from the first referred pain, ranged between 12 to $48 \mathrm{~h}$ and ended in caesarean section in four cases. The mothers reported having experienced delays at the health facilities, particularly in making the decision to perform operative delivery. One mother stayed for 2 weeks at the maternity waiting home and the day the labour started it took $12 \mathrm{~h}$ before she was referred to the hospital. Two mothers arrived at the primary health facility with minor pain and one stayed for 4 days and the other 1 week, before the decision to transfer was made. The mother who stayed for 1 week was told at the moment of the transfer that the baby was not alive and was in pelvic presentation. An ambulance was used to transfer four mothers to the next level of care, while one family had to hire a taxi.

During labour, all women were supported by a nurse and the caesarean sections were performed by non-physician clinicians [36]. All the caesareans but one were performed at the same district hospital in Gaza Province.

All of the babies died; four were stillborn, and one died very soon after birth. All mothers rated their previous labour as being very difficult. None of them felt they had been humiliated or treated disrespectfully and no one reported any physical abuse. All four who had had a caesarean section felt that the intervention was necessary.

\section{Fistula onset and treatment}

Four mothers noticed the onset of the urine leakage one-to-two days after the caesarean section was performed; the only patient who had had a vaginal delivery noticed it 5 days after. The complications were recognized at the hospital, but no surgical treatment was proposed to them. All agreed that suffering a fistula is a big problem and none of the mothers were aware that fistula is treatable. Their overall health was perceived as good, except for one mother, who said it was neither good nor bad. Only one of the two, who referred to the abdominal pain as "terrible", had received any treatment. None of the mothers had had intercourse since the appearance of the fistula.

During this study, fistula repair was offered to all of the mothers diagnosed with fistula. One mother, however, had a contraindication for surgery (receiving treatment for psychiatric complications) and was hence not referred for surgical repair. All of the remaining four fistulae were successfully closed. Of the three mothers who came for follow-up, one reported urinary incontinence, but there was no breakdown in the surgical repair.

\section{Discussion}

Main findings

Our findings indicate a community-based incidence of obstetric fistula of 1.1 per 1000 recently pregnant women. Caesarean section (CS) was performed for all but one of the deliveries causing the fistulae. Distance and access to the health care facility does not seem to be a reason for the delay in receiving the emergency caesarean section. All of the mothers identified and reached the primary health facility in reasonable time. A Type 3 


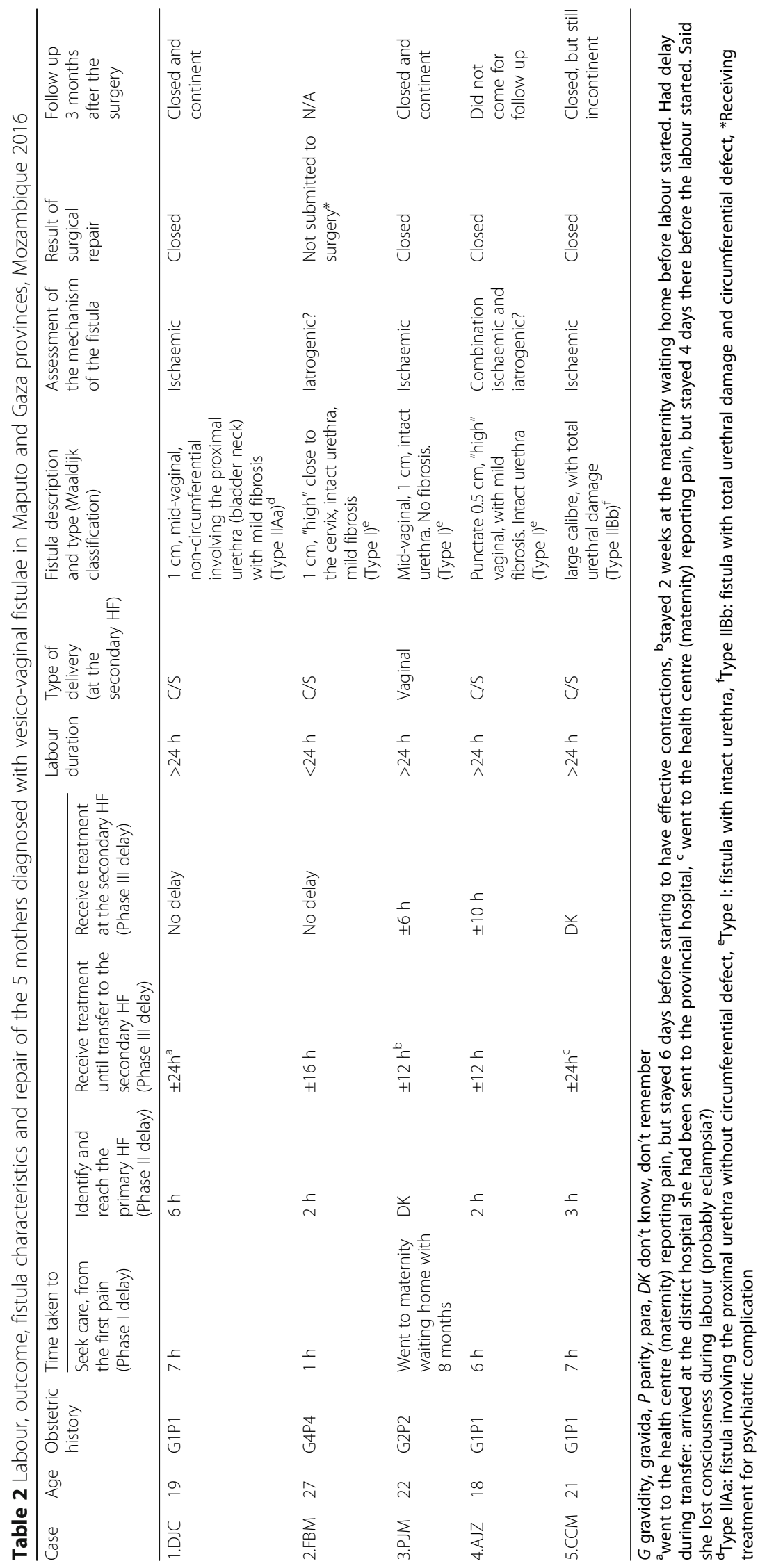


phase of delay occurred in the management of these cases: delay in diagnosing obstructed labour, deciding the need of a CS, and the transferring of the patient to a higher level hospital. All but one woman with fistulae were referred for surgical repair and the fistulae were successfully closed. Incontinence persisted in one case after 3 months.

\section{Strengths and limitations}

The major strength of our study is the community-based identification of the suspected cases (with reported symptoms) combined with hospital-based clinical examination, which maximised the likelihood of accurate case identification. The population of recently pregnant women was identified from a well-identified cohort of pregnant women and was followed over a defined period of time, minimising the risk of selection bias. Detection bias was minimized by the use of a standardized data collection instrument (fistula module) together with clinical examination.

Although nondisclosure of incontinence symptoms in the questionnaire interview was possible, it seems unlikely, given that the data collectors were trained to explain the meaning of urinary incontinence and to explain that treatment would be offered free of charge to those detected and to emphasise that all the information collected would be kept confidential. A risk of underestimating the number of fistula cases should be considered, as two of the 30 mothers who reported incontinence symptoms did not attend for subsequent examination. The ostracism and stigma associated with the condition [37] might have deterred the interviewed mothers from disclosing their symptoms due to the risk of being excluded from their households and/or communities. A further limitation was that we had no information about the pre- or post-operative circumstances.

\section{Interpretation}

The fistula incidence of 1.1 per 1000 recently pregnant women that we found is higher than Adler's [11] pooled incidence estimates in LMICs of 0.09 and 0.66 per 1000 recently pregnant women in community-based and hospital-based studies, respectively. It is recognized in the literature that reported rates of obstetric fistula vary widely. Taking into account that our survey was followed by gold-standard gynaecological exams, the variation could represent true differences in incidence. We emphasize the importance of the clinical examination and confirmation of all women who reported fistula symptoms: $78 \%$ of the mothers who reported fistula-like symptoms had conditions other than fistula. Similar to our study, a community-based screening for fistulae in Nigeria, using the fistula module questionnaire, found that $53 \%$ of the women who reported symptoms did not have a fistula [38].

Our estimate is close to the fistula incidence of 1.2 per 1000 births found in the only prospective populationbased study, which was undertaken in rural West Africa in 1999 [12]. That incidence was, similar to our study, observed in rural areas where women are at higher risk of labour complications. However, the study from West Africa was undertaken in an area with a much lower proportion of births attended by skilled health personnel $(39.6 \%$ vs $85 \%)$ and lower caesarean section rates $(0.7 \%$ vs $3.8 \%)$. Although the low caesarean section rate could suggests an unmet need $[39,40]$, it is important to consider that most of the identified cases in this study occurred amongst women who arrived in a timely manner at the primary health facility (except one who took $13 \mathrm{~h}$ ). Thus, while several studies have described delays in deciding to seek care (first delay) and accessing a health facility (second delays) as being important factors in fistula formation [41-43], we found most delays occurring at health facilities (third delay). Indeed, the detection of high-risk deliveries and decision to refer women to a hospital with surgery capability was not timely. In addition, delays in reaching the referral hospital, mostly due to unavailability of ambulances, have been observed, similar to those described by Waiswa et al. (2017) [44] from Southern Tanzania, where there are no ambulances at the primary facility level, which is why emergency transport is a constraint. These findings that the third delay (receiving inadequate care at health facility) contributed to obstetric problems are corroborated by other studies conducted in Tanzania, Gambia and elsewhere $[45,46]$. We have classified the failure to detect obstetric problems in a timely manner at the primary health facility as a third delay, according to the model described by Thaddeus and Maine (1994) [33] and Berhan and Berhan (2014) [47]. However, as discussed by Gabrysh and Campbell (2009) [48], these delays can also be classified as second emergency delays, when complications that cannot be managed at the primary facility require referral to a higher-level facility. Whatever the classification, we consider that the issue is the capacity of the health system in caring for the women in labour.

Prolonged obstructed labour is the most common cause of fistula formation [2, 49, 50]. Three of the five identified fistulae were probably ischaemic in origin (two type II fistula and one type I). However, in two cases, the type I fistulae were high vaginal, close to the cervix. This type of fistula (equivalent to type 1 on the Goh classification [51], i.e., more than $3.5 \mathrm{~cm}$ from the external urinary meatus) is presumed to be iatrogenic in aetiology [5]. This could be the case for the type I fistula, which occurred where the duration of labour was less than 
$24 \mathrm{~h}$. A combination of ischaemic and iatrogenic mechanisms could be suggested for the other type I, where the duration of labour was $30 \mathrm{~h}$. It is important to mention that, for a type I fistula to be considered as iatrogenic, it would either mean that the incision of the uterus was too extensive or that the sutures used for the closure of the uterus would have included the fundus or posterior wall of the bladder and would have to be extended to the vesico vaginal septum. Although the mechanism involved could not be verified in this study, there are reports of an increasing proportion of iatrogenic urogenital fistulae in LMICs, primarily in obstetric interventions, especially caesarean section $[5,52]$, and it is possible that this was the case in our study. This calls for more quality of care research on obstructed labour and caesarean delivery.

All four foetuses delivered by caesarean section were stillborn. We do not have information about the circumstances of the management of these cases, but, considering that some of these could have been obstructed labour with foetal death, we should consider whether craniotomy may have been an alternative method of delivering these babies. This procedure is performed in LMICs [53-55] and is especially useful in patients who come from rural areas and run the risk of the complications of an abdominal-route delivery and the risks of rupture of uterine scar during subsequent pregnancies or labour $[56,57]$.

Although the fistulae were recognized at the health facilities, no treatment was proposed to these mothers at the time. This could be due to the limited availability of fistulae repair services and a lack of fistulae surgeons, which was reported in several settings in sub-Saharan Africa, including Mozambique, where at least $80 \%$ of women with fistula are estimated to have no access to fistulae repair each year [58]. However, the lack of adequate postpartum assessment and referral of the women with fistulae should be also considered.

This is the first study in Mozambique estimating fistula incidence in rural areas through a community-based survey with suspected obstetric fistulae being examined and treated at a health facility. Our results could be an underestimate for the country's epidemiology as a whole, considering that our study area has a high density of health facilities compared to other parts of the country where more first and second delays would be expected.

\section{Conclusion}

This population-based study reports a high incidence of obstetric fistulae in an area with a high rate of facility births. The most frequent delay was that of receiving care once at a health facility as opposed to delays relating to deciding to seek care or reaching the health facility. This raises concerns regarding the quality of care provided to women during delivery. Furthermore, our results suggest the need to improve postpartum care, especially for early identification and adequate management of obstetric fistulae. Future studies, particularly those that focus on quality of care in obstructed labour, use of caesarean delivery and role of alternative options such as craniotomy in cases of foetal death to avoid iatrogenic fistula, should be considered.

\section{Additional file}

Additional file 1: Health survey with women having delivered during the last year. (PDF $835 \mathrm{~kb}$ )

\section{Abbreviations}

CISM: Manhiça Health Research Center; CLIP: Community Level Interventions for Pre-eclampsia; CS: Caesarean section; DHS: Demographic and Health Survey; IDI: In-Depth Interview; LMIC: Low and Middle Income countries; UEM: Eduardo Mondlane University; UNFPA: United Nation Population Fund; WHO: World Health Organisation

\section{Acknowledgements}

We thank the women participating in this study for their commitment, the CISM for the logistic support and the Manhiça District Hospital for their assistance. We thank the field workers, the supervisors, the assistant manager Rogério Chiau, the nurses Analisa Matavele, Florencia Cherinda, Dulce Mulungo and the CLIP study team in Mozambique (Charfudin Sacoor, Anifa Valá, Salésio Macuacua, Helena Boene) and the CLIP working group at UBC (Beth Payne, Marianne Vidler, Sumedha Sharma, Jing (Larry) Li, Tang Lee, Domena Tu, Alison Dube, Jeffrey Bone, Dustin Dunsmuir, Meera Madhavan) for their commitment and dedication. Thanks also to Dr. Igor Vaz and his team at the Urology Service and Dr. Elvira Luis and her team at the Obstetrics and Gynaecology Department in Maputo Central Hospital, for caring for the patients and performing the surgeries.

\section{Funding}

The Program of Research Cooperation between Sweden Universities and Eduardo Mondlane University in Mozambique (UEM) (Sida decision 2011 002102, No 51140011) financially supported this study. The CLIP trial is part of the University of British Colombia PRE-EMPT (Pre-eclampsia/Eclampsia Monitoring Prevention and Treatment), a project at University of British Columbia Vancouver, funded by the Bill and Melinda Gates Foundation.

\section{Availability of data and materials}

The datasets used and analysed during this study will be stored at the CISM repository and are available by request to the corresponding author after following the CISM policy on data sharing.

\section{Authors' contributions}

$\mathrm{SM}, \mathrm{ES}, \mathrm{CH}, \mathrm{AB}$ and $\mathrm{UH}$ designed the study. SM, HB, KM and ES had primary responsibility for the data acquisition. SM had primary responsibility for analysis and interpretation of the data and for writing the manuscript. All authors, SB, ES, CH, AB, HB, PVD, KM and UH, participated in the analysis, interpretation of the study results, revision of the manuscript, and input to various drafts. All authors read and approved the final manuscript.

\section{Ethics approval and consent to participate}

Ethical approval was provided by the Bioethics Review Joint Committee of the UEM Faculty of Medicine and Maputo Central Hospital (CIBS FM\&HCM) 33/2015, dated 28 August 2015). Informed consent was obtained from each participant before interview. Those aged less than 18 years were asked about their willingness to participate. Thereafter, informed consent was obtained from their parents or legal guardians, and additional assent was then obtained from those participants. For illiterate participants, a literate witness was involved in the consent process, the participant's fingerprint was taken, and the consent form signed by the witness and the data collector. Women affected by obstetric fistula who presented themselves because of their awareness of the study but who did not fulfil the study inclusion criteria, were offered treatment. 


\section{Consent for publication}

Not applicable.

\section{Competing interests}

The authors declare that they have no competing interests.

\section{Publisher's Note}

Springer Nature remains neutral with regard to jurisdictional claims in published maps and institutional affiliations.

\section{Author details}

'Department of Obstetrics and Gynaecology, Faculty of Medicine, Universidade Eduardo Mondlane (UEM), Av. Agostinho Neto 679, 1100 Maputo, Mozambique. 'Department of Women's and Children's Health, Uppsala University, Akademiska sjukhuset, SE-75185 Uppsala, Sweden. ${ }^{3}$ Department of Public Health Sciences, Karolinska Institutet, Tomtebodavagen 18A, Plan 4, Stockholm, Sweden. ${ }^{4}$ Department of Disease Control, London School of Hygiene and Tropical Medicine, Keppel St, London WC1E 7HT, UK. ${ }^{5}$ Centro de Investigação em Saúde de Manhiça (CISM), Rua 12, Vila da Manhiça, 1121 Manhiça, Mozambique. ${ }^{6} \mathrm{Global}$ Women's Health, King's College, London, UK. `University College London, Institute for Global Health, Gower St, London WC1E 6BT, UK. ${ }^{8}$ Department of Public Health, Faculty of Medicine, UEM, Av. Salvador Allende 702 R/C, Maputo, Mozambique. ${ }^{9}$ Department of Physiological Science, Clinical Pharmacology, Faculty of Medicine, UEM, Av. Salvador Allende 702 R/C, Maputo, Mozambique.

Received: 27 July 2017 Accepted: 31 October 2017

Published online: 10 November 2017

\section{References}

1. WHO. Obstetric fistula: Guiding principles for clinical management and program development. Geneva: World Health Organization; 2006.

2. De Ridder D, Badlani GH, Browning A, et al. Fistulas in the developing world. In: Abrams P, Cardozo L, Khoury S, Wein A, editors. Incontinence, 4th ed. Paris: Health Publications Ltd.; 2009. p. 1419-58.

3. Neilson JP. Obstructed labor. Br Med Bull. 2003;67(1)

4. Wall $L L$, et al. The obstetric vesicovaginal fistula in the developing world Obstet Gynecol Surv. 2005;60(7 Suppl 1):S3-S51. https://www.ncbi.nIm.nih. gov/m/pubmed/16034313/?i=4\&from=/16895244/related.

5. Hilton P. Trends in the aetiology of urogenital fistula: a case of 'retrogressive evolution'? Int Urogynecol J. 2016;27(6):831-7.

6. Arrowsmith SD. Genitourinary reconstruction in obstetric fistulas. J Urol. 1994;152:403-6.

7. Cowgill KD, et al. Obstetric fistula in low-resource countries: an under-valued and under-studied problem-systematic review of its incidence, prevalence, and association with stillbirth. BMC Pregnancy Childbirth. 2015;15:193.

8. Wall LL. Obstetric vesicovaginal fistula as an international public-health problem. Lancet. 2006;368(9542):1201-9.

9. Zheng AX, Anderson FW. Obstetric fistula in low-income countries. Int J Gynaecol Obstet. 2009;104(2):85-9.

10. Stanton C, Holtz SA, Ahmed S. Challenges in measuring obstetric fistula. Int J Gynaecol Obstet. 2007;99(Suppl 1):S4-9.

11. Adler AJ, et al. Estimating the prevalence of obstetric fistula: a systematic review and meta-analysis. BMC Pregnancy Childbirth. 2013;13:246.

12. Vangeenderhuysen C, Prual A, Ould D, Joud E. Obstetric fistulae: incidence estimates for sub-Saharan Africa. Int J Gynaecol Obstet. 2001;73(1):65-6.

13. Ferdous J, et al. Occurrence and determinants of postpartum maternal morbidities and disabilities among women in Matlab, Bangladesh. J Health Popul Nutr. 2012;30(2):143-58

14. Fronczak $\mathrm{N}$, et al. Delivery-related complications and early postpartum morbidity in Dhaka, Bangladesh. Int J Gynaecol Obstet. 2005;91(3):271-8.

15. Filippi $\mathrm{V}$, et al. Health of women after severe obstetric complications in Burkina Faso: a longitudinal study. Lancet. 2007:370(9595):1329-37.

16. Filippi $\mathrm{V}$, et al. Effects of severe obstetric complications on women's health and infant mortality in Benin. Tropical Med Int Health. 2010;15(6):733-42.

17. Prual A, et al. Severe obstetric morbidity of the third trimester, delivery and early puerperium in Niamey (Niger). Afr J Reprod Health. 1998;2(1):10-9.

18. Tuncalp $\mathrm{O}$, et al. Measuring the incidence and prevalence of obstetric fistula: approaches, needs and recommendations. Bull World Health Organ. 2015; 93(1):60-2.
19. Biadgilign S. A population based survey in Ethiopia using questionnaire as proxy to estimate obstetric fistula prevalence: results from demographic and health survey. Reprod Health. 2013;10:14.

20. Jokhio $\mathrm{AH}$, et al. Prevalence of obstetric fistula: a population-based study in rural Pakistan. BJOG. 2014;121(8):1039-46.

21. De Ridder D, Badlani $\mathrm{GH}$, Browning A, et al. Fistulas in the developing world. In: Abrams P, Cardozo L, Khoury S, Wein A, editors. Incontinence, 4th ed. Paris: Health Publications Ltd.; 2009. p. 1419-58. https:/www.ics.org/committees/ developingworld/professionaleducation/internationalconsultationonincontinence.

22. Abreu RD, Varregoso J, Vaz I, Gomes C. Vesico Vaginal Fistulae: Mozambique, an overview. Rev Clin Hosp Prof Dr Fernando Fonseca. 2015; 3(2):27-32.

23. Ngongo C, et al. What to measure and why? Experience developing monitoring indicators for an emerging maternal health issue: the case of obstetric fistula. J Health Inform Dev Countries. 2015:9(1):14-22. http://www. jhidc.org/index.php/jhidc/article/view/133/175

24. Khowaja AR, et al. The feasibility of community level interventions for preeclampsia in South Asia and sub-Saharan Africa: a mixed-methods design. Reprod Health. 2016:13(Suppl 1):56.

25. UNFPA. UNFPA Proposal for Obstetric Fistula, The Demographic and Health Surveys Program Revising DHS-7 Questionnaires. 2014. Retrieved from https://userforum.dhsprogram.com/index.php?t=getfile\&id=217\&S=Google.

26. WHO. Responsiveness Questionnaires. 2001 [cited 2016 February, 16]; available from: http://www.who.int/responsiveness/surveys/en/. Accessed 4 Nov 2017.

27. Colbourn T, et al. Cost-effectiveness and affordability of community mobilisation through women's groups and quality improvement in health facilities (Maikhanda trial) in Malawi. Cost Eff Resour Alloc. 2015:13(1):1.

28. Colbourn T, et al. Effects of quality improvement in health facilities and community mobilization through women's groups on maternal, neonatal and perinatal mortality in three districts of Malawi: MaiKhanda, a cluster randomized controlled effectiveness trial. Int Health. 2013:5(3):180-95.

29. Raja A. Android and ODK base d data collection framework to aid in epidemiological analysis. Online Journal of Public Health Informatics. 2014;5(3):228

30. Brunette W, et al. Open Data Kit 2.0: Expanding and Refining Information Services for Developing Regions. In Hotmobile13 14th Workshop on Mobile Computing Systems and Applications. Jekyll Island, Georgia: ACM Digital Library; 2013.

31. Waaldijk K. Surgical classification of obstetric fistulas. Int J Gynaecol Obstet. 1995:49(2):161-3.

32. Capes $T$, et al. Comparison of two classification systems for vesicovaginal fistula. Int Urogynecol J. 2012;23(12):1679-85.

33. Thaddeus S, Maine D. Too far to walk: maternal mortality in context. Soc Sci Med. 1994:38(8):1091-110

34. Harris PA, et al. Research electronic data capture (REDCap)-a metadatadriven methodology and workflow process for providing translational research informatics support. J Biomed Inform. 2009;42(2):377-81.

35. R Core Team, R: A language and environment for statistical computing 2016, R Foundation for Statistical Computing: Vienna, Austria.

36. Mullan F, Frehywot S. Non-physician clinicians in 47 sub-Saharan African countries. Lancet. 2007;370(9605):2158-63.

37. Weston $\mathrm{K}$, et al. Depression among women with obstetric fistula in Kenya. Int J Gynaecol Obstet. 2011;115(1):31-3.

38. Tuncalp $\mathrm{O}$, et al. Community-based screening for obstetric fistula in Nigeria: a novel approach. BMC Pregnancy Childbirth. 2014;14:44.

39. Chu K, et al. Cesarean section rates and indications in sub-Saharan Africa: a multi-country study from Medecins sans Frontieres. PLoS One. 2012;7(9): e44484.

40. Long $Q$, et al. Caesarean section rates in Mozambique. BMC Pregnancy Childbirth. 2015;15:253.

41. Miller S, et al. Obstetric fistula: a preventable tragedy. J Midwifery Womens Health. 2005;50(4):286-94.

42. Barnes-Josiah D, Myntti C, Augustin A. The "three delays" as a framework for examining maternal mortality in Haiti. Soc Sci Med. 1998;46(8):981-93.

43. Hussein AK, Mpembeni R. Recognition of high risk pregnancies and referral practices among traditional birth attendants in Mkuranga District, coast region. Tanzania Afr J Reprod Health. 2005;9(1):113-22.

44. Waiswa $P$, et al. Effects of the EQUIP quasi-experimental study testing a collaborative quality improvement approach for maternal and newborn health care in Tanzania and Uganda. Implement Sci. 2017;12(1):89. 
45. Cham M, Sundby J, Vangen S. Maternal mortality in the rural Gambia, a qualitative study on access to emergency obstetric care. Reprod Health. 2005;2(1):3.

46. Mselle L. Waiting for attention and care: birthing accounts of women in rural Tanzania who developed obstetric fistula as an outcome of labour. BMC Pregnancy and Childbirth. 2011;11(1):75.

47. Berhan Y, Berhan A. Commentary: reasons for persistently high maternal and perinatal mortalities in Ethiopia: part II-perspective of the "three delays" model. Ethiop J Health Sci. 2014;24(Suppl):137-48.

48. Gabrysch S, Campbell OM. Still too far to walk: literature review of the determinants of delivery service use. BMC Pregnancy Childbirth. 2009;9:34.

49. Ramphal S, Moodley J. Vesicovaginal fistula: obstetric causes. Curr Opin Obstet Gynecol. 2006;18(2):147-51.

50. Hillary CJ, et al. The Aetiology, treatment, and outcome of urogenital fistulae managed in well- and low-resourced countries: a systematic review. Eur Urol. 2016:70(3):478-92.

51. Goh JT. A new classification for female genital tract fistula. Aust N Z J Obstet Gynaecol. 2004:44(6):502-4.

52. Raassen TJ, Ngongo CJ, Mahendeka MM. latrogenic genitourinary fistula: an 18-year retrospective review of 805 injuries. Int Urogynecol J. 2014;25(12): 1699-706.

53. Zeidan A, Abdella A. Destructive vaginal deliveries at a teaching hospital in Addis Ababa. Ethiopia Ethiop Med J. 2007:45(1):39-45.

54. Maharaj D, Moodley J. Symphysiotomy and fetal destructive operations. Best Pract Res Clin Obstet Gynaecol. 2002;16(1):117-31.

55. Gupta U, Chitra R. Destructive operations still have a place in developing countries. Int J Gynaecol Obstet. 1994:44(1):15-9.

56. Singhal SR, et al. Destructive operations in modern obstetrics. Arch Gynecol Obstet. 2005;273(2):107-9.

57. Sikka P, et al. Destructive operations-a vanishing art in modern obstetrics: 25 year experience at a tertiary care center in India. Arch Gynecol Obstet. 2011;283(5):929-33.

58. UNFPA,EngenderHealth. Obstetric Fistula Needs Assessment Report: Findings from Nine African Countries. New York: United Nations Population Fund; 2003. [Cited 2017 April, 20]; available from http://www.unfpa.org/ sites/default/files/pub-pdf/fistula-needs-assessment.pdf.

\section{Submit your next manuscript to BioMed Central} and we will help you at every step:

- We accept pre-submission inquiries

- Our selector tool helps you to find the most relevant journal

- We provide round the clock customer support

- Convenient online submission

- Thorough peer review

- Inclusion in PubMed and all major indexing services

- Maximum visibility for your research

Submit your manuscript at www.biomedcentral.com/submit
) Biomed Central 